\title{
Virtual Screening of Compounds by Chemoinformatics Tools in the Chemistry Labs ${ }^{+}$
}

\author{
Amalia Stefaniu 1,*, Lucia Pirvu 1, Eleonora-Mihaela Ungureanu 2, Liviu Birzan ${ }^{3}$, \\ Gabriela Stanciu ${ }^{4}$, Laura-Bianca Enache ${ }^{2}$ and Veronica Anastasoaie ${ }^{2}$
}

1 National Institute for Chemical, Pharmaceutical Research and Development, ICCF, 112 Vitan av., 031299 Bucharest, Romania; lucia.pirvu@yahoo.com

2 Department of Inorganic Chemistry, Physical Chemistry and Electrochemistry, "Politehnica" University of Bucharest, Gheorghe Polizu 1-7, 011061 Bucharest, Romania; em_ungureanu2000@yahoo.com (E.-M.U.); laurabianca2794@gmail.com (L.-B.E.); veronyk_92@yahoo.com (V.A.)

3 Institute of Organic Chemistry “C. D. Nenitzescu” of Romanian Academy, Splaiul Independentei 202B, PO Box 35-108, 060023 Bucharest, Romania; lbirzan@yahoo.com

4 Department of Chemistry and Chemical Engineering, Ovidius University of Constanta, 124 Mamaia Blvd, 900527 Constanta, Romania; gstanciu66@yahoo.com

* Correspondence: astefaniu@gmail.com

† Presented at the Virtual Eurachem Workshop 2020 - “Quality Assurance for Analytical Laboratories in the University Curriculum”, 14-15 July 2020; Available online: https://eurachem2020.ro/.

Published: 22 September 2020

\begin{abstract}
Virtual screening of compound libraries for property predictions has various applications such as: prediction of oxido-reduction potentials in view of molecular recognition, drug-likeness assessment, and design of new potential therapeutic agents, quantitative structure-property and activity relationships (QSPR/QSAR) modeling to design new structures and property predictions. Available free online platforms or specialized software provide us with vital help to design, characterize and evaluate molecular features and descriptors for imaginary systems or newly synthesized ones, to establish their potential for new applications by controlling and modeling their chemical/ biochemical behavior and properties. Predictions allow us to reduce the time spent, the cost of reagents or elaborate and costly assays, and provide us enlarged and complex perspectives.
\end{abstract}

Keywords: chemoinformatics; prediction property; drug-likeness assessment; electrochemistry behavior

\section{Introduction}

In the early development of the drug discovery process, the researchers of the medicinal chemistry field, explore chemical and biology information relevant for a new chemical entity to become a successful, marketable drug. They study its physico-chemical properties and molecular features, its behavior under various conditions and interactions with biological targets. Often, such screening research is accompanied by computational and predictive models, such as molecular docking and molecular mechanic simulations studies [1,2], conducting to lead discovery and optimization.

Aiming to facilitate faster and rational drug design, the pharma researchers have imposed some limitations on the values of chemical key molecular descriptors for drug candidates. Thus, for good oral bioavailability, the Lipinski's rule of five (RO5) [3,4] developed at Pfizer, states that a molecule should not exceed 500 Dalton in molecular weight, the water-octanol partition coefficient should be less than 5 , the number of hydrogen donor groups $<5$, and the number of hydrogen acceptor groups $<10$. These parameters generally dictate the absorption and permeation of a drug. The hydrophilic- 
lipophilic character of compounds can be evaluated by the prediction of aqueous solubility expressed as $\log$ units of molar solubility ( $\mathrm{mol} / \mathrm{L})$, or $\log S$ and its hydrophobicity established from molecular structures features. Geometric molecular descriptors, such as area, volume, and ovality, or parameters derived from charge distribution, such as dipole moment, Mulliken charges, or electronegativity, are easily predicted using various computational tools that perform calculations on optimized geometries of structures using molecular mechanics force fields. An example of such software is Spartan Software, from Wavefunction Inc, Irvine, CA, USA [5], that uses a variety of hybrid functional with high accuracy of prediction. Veber and co-workers [6] supplement these criteria with a limitation for polar surface area (no larger than $140 \AA^{2}$ ) and the number of rotatable bonds (maximum 10) for good oral bioavailability.

Molinspiration (https://www.molinspiration.com) is a free online platform that allows users to calculate properties required for drug-likeness assessment and also bioactivity scores reported to common human target proteins (for GPCR ligands, ion channel modulators, kinase inhibitors, and nuclear receptor ligands), with the possibility to screen extensive compound library and to separates those with good drug-likeness from inactive ones.

Thus, researchers in medicinal and pharma fields, who now benefit from advances chemoinformatics tools, can now scan thousands of compounds to start from their known structure to evaluate drug potential.

For electrochemistry applications, in efforts to detect the limits of detection for heavy metals, by complexation, an important preliminary step for the choice of ligand to be synthesized, characterized, and studied by electrochemical measurements, can be considered the prediction of oxido-reduction potentials from calculated frontier molecular orbitals (FMOs) energy diagram. The results of computations for the energy of the Highest Occupied Molecular Orbital (HOMO) and of the Lowest Unoccupied Molecular Orbital (LUMO) can be associated with the ionization potential and electron affinity, respectively, according to Koopmans' theorem [7], related to global molecular reactivity parameters evaluation.

\section{Theoretical Models and Relationships}

\subsection{Global Reactivity Parameters}

Kinetic stability and reactivity of molecules can be easily assessed using formulas stated in Koopmans' theorem [8], as follows: ionization potential $\left(I=-E_{\text {номо }}\right)$, electron affinity $\left(A=-E_{\mathrm{Lumo}}\right)$, electronegativity $(\chi=(I+A) / 2)$, global hardness $(\eta=(I-A) / 2)$, local softness $(\sigma=1 / \eta)$, chemical potential $(\mu=($ Еномо + ELuмо $) / 2)$ and global electrophilicity index $\left(\omega=\mu^{2} / 2 \eta\right)$.

\subsection{Local Reactivity Parameters}

Atomic charges, atomic electrostatic potential, and atomic Fukui indices can be predicted using density functional theory (DFT) or Möller-Plesset methods (MP2). Petkova and co-workers [8] found the correlation of these computed parameters with the experimentally measured XPS binding energies for some coumarin derivatives.

\subsection{Redox Potentials}

In electrochemistry, the DFT calculations of energy levels for frontier molecular orbitals can be correlated with the oxidation and reduction potentials, useful to assess the complexation ability of some ligands with heavy metals ions as $\mathrm{Pb}$ (II), $\mathrm{Cd}(\mathrm{II}), \mathrm{Cu}(\mathrm{II})$ or $\mathrm{Hg}(\mathrm{II})$, and to design selective sensors, as we reported in our previous work [9].

The loss of an electron from the Highest Occupied Molecular Orbital (HOMO) corresponding to the oxidation process, is associated with the ionization energy. The electron affinity, suggested by the LUMO energetical level, is correlated with the reduction state given by the gain of an electron and the energy released when an electron is added to the LUMO of a neutral molecule in the gaseous state). 
$I=-$ Еномо ; $A=-$ Eцuмо. In the empirical equations of Bredas et al. [10,11], the energy of frontier molecular orbitals is linked to the oxido-reductions potentials, including the ferrocene value of -4.4 $\mathrm{eV}$, thus:

$$
\begin{aligned}
& E_{\text {номо }}=-\mathrm{e}\left[E_{\text {ox }}{ }^{\text {onset }}+4.4\right] \\
& E_{\text {LUMO }}=-\mathrm{e}\left[E_{\text {red }}{ }^{\text {onset }}+4.4\right]
\end{aligned}
$$

Moreover, other authors [12,13] relate the electrochemical potentials to the vacuum level to electrochemical measurements:

$$
\begin{aligned}
& I=-\left(E_{\mathrm{ox}}+4.14\right) \mathrm{eV} \\
& E=-\left(E_{\text {red }}+4.14\right) \mathrm{eV}
\end{aligned}
$$

where:

$E_{\text {ox }}$ and $E_{\text {red }}$ are the onset potentials of the oxidation and reduction waves relative to an Ag 0.01 $\mathrm{M} / \mathrm{AgNO}_{3}$ reference electrode; 4.14 represents the difference between the vacuum level potential of the normal hydrogen electrode $(\mathrm{NHE}=4.44 \mathrm{eV})$ and the potential of the $\mathrm{Ag} / \mathrm{AgNO}_{3}$ electrode.

In some studies, a linear relationship exists between the molecular orbital energies and their reduction and oxidation potentials obtained from cyclic voltammetry. These linear correlations can be used to estimate the redox potentials of unknown complexes, using the calculated HOMO and LUMO energy levels. J. Conradie and co-workers [14] found linear relations between the experimentally measured reduction potential of a series of some $\beta$-diketones and their calculated LUMO energies and electron affinity, and a linear relationship between oxidation potential and calculated HOMO energies and ionization potential, respectively.

In fact, the approximate linear relationship between HOMO and LUMO energies and oxidation/reduction potentials can be deduced from the Marcus quadratic relationship for interfacial electron transfer [15]:

$$
\text { EOX/RED }=\mathrm{a}+\mathrm{b} \cdot \text { Еномо/Luмо }
$$

where EOx/RED are the experimental redox potentials; Еномо/Luмо are the calculated HOMO and LUMO energies; and $\mathrm{a}$ and $\mathrm{b}$ are constants.

\section{Conclusions}

Models based on prediction for the estimation of various molecular properties aiming to design compounds for enhanced applicability in multiple domains such as medicine, food and nutraceutical industries, electrochemistry, sensors, and metal detections, etc., are widely used as preliminary and predictive studies. Chemoinformatics tools in chemistry and multidisciplinary approaches become useful instruments in education, and we must raise awareness among students about their use and benefits, and include such valuable resources in the educational process.

Author Contributions: Conceptualization, A.S., and L.B.; methodology, A.S.; software, A.S.; validation, E.-M.U., and G.S.; formal analysis, L.B.; investigation, L.-B.E., and V.A.; resources, L.B. and A.S. data curation, L.P.; writing-original draft preparation, A.S.; writing-review and editing, A.S. and L.B.; visualization, E.-M.U.; supervision, E.-M.U.; project administration, L.P.; funding acquisition, L.P. All authors have read and agreed to the published version of the manuscript.

Funding: This work was supported by the ANCSI program POC-A1-A1.2.3-G-2015, Project title "New technologies and natural derived products for human health use", Contract no.60/05.09.2016, ID P_40_406, SMIS 105542.

Conflicts of Interest: The authors declare no conflict of interest. 


\section{References}

1. Pirvu, L.; Stefaniu, A.; Neagu, G.; Albu, B.; Pintilie, L. In Vitro Cytotoxic and Antiproliferative Activity of Cydonia oblonga flower petals, leaf and fruit pellet ethanolic extracts. Docking simulation of the active flavonoids on anti-apoptotic protein Bcl-2. Open Chem. 2018, 16, 591-604.

2. Stefaniu, A. Introductory Chapter: Molecular Docking and Molecular Dynamics techniques to achieve rational drug design. In Molecular Docking and Molecular Dynamics; Stefaniu, A., Ed., IntechOpen: London, UK, 2019; pp. 1-3.

3. Lipinski, C.A. Lead- and drug-like compounds: The rule-of-five revolution. Drug Discov. Today Technol. 2004, 1, 337-341.

4. Lipinski, C.A.; Lombardo, F.; Dominy, B.W.; Feeney, P.J. Experimental and computational approaches to estimate solubility and permeability in drug discovery and development settings. Adv. Drug Deliv. Rev. 2001, 46, 3-26.

5. Shao, Y.; Molnar, L.F.; Jung, Y.; Kussmann, J.; Ochsenfeld, C.; Brown, S.T.; Gilbert, A.T.; Slipchenko, L.V.; Levchenko, S.V.; O'Neill, D.P.; et al. Advances in methods and algorithms in a modern quantum chemistry program package. Phys. Chem. Chem. Phys. 2006, 8, 3172-3191.

6. Veber, D.F.; Johnson, S.R.; Cheng, H.Y.; Smith, B.R.; Ward, K.W.; Kopple, K.D. Molecular properties that influence the oral bioavailability of drug candidates. J. Med. Chem. 2002, 45, 2615-2623.

7. Koopmans, T. Über die Zuordnung von Wellenfunktionen und Eigenwerten zu den Einzelnen Elektronen Eines Atoms. Physica 1934, 1, 104-113.

8. Petkova, N.I.; Nikolova, R.D.; Kostov, K.L.; Mineva, T.; Vayssilov, G.N. Theoretical and Experimental Local Reactivity Parameters of 3-substituted Coumarin Derivatives. J. Phys. Chem. A 2014, 118, 11062-11073.

9. Stefaniu, A.; Pop, M.-D.; Arnold, G.-L.; Birzan, L.; Pintilie, L.; Diacu, E.; Ungureanu, E.-M. DFT calculations and electrochemical studies on azulene ligands for heavy metal ions detection using chemically modified electrodes. J. Electrochem. Sci. Eng. 2018, 1, 73-85.

10. Bredas, J.L.; Silbey, R.; Boudreux, D.S.; Chance, R.R. Chain-length dependence of electronic and electrochemical properties of conjugated systems: Polyacetylene, polyphenylene, polythiophene, and polypyrrole. J. Am. Chem. Soc. 1983, 105, 6555-6559.

11. Leonat, L.; Sbârcea, G.; Brânzoi, I.V. Cyclic voltammetry for energy levels estimation of organic materials. U.P.B. Sci. Bull. B 2013, 75, 111-118.

12. Kucur, E.; Riegler, J.; Urban, G.A.; Nann, T. Determination of quantum confinement in CdSe nanocrystals by cyclic voltammetry. J. Chem. Phys. 2003, 119, 2333-2337.

13. Brus, L.E. A simple model for the ionization potential, electron affinity, and aqueous redox potentials of small semiconductor crystallites. J. Chem. Phys. 1983, 79, 5566-5571.

14. von Eschwege, K.G.; Conradie, J. Redox Potentials of Ligands and Complexes-A DFT Approach. S. Afr. J. Chem. 2011, 64, 203-209.

15. Méndez-Hernández, D.D.; Tarakeshwar, P.; Gust, D.; Moore, T.A.; Moore, A.L.; Mujica, V. Simple and Accurate Correlation of Experimental Redox Potentials and DFT-calculated HOMO/LUMO Energies of Polycyclic Aromatic Hydrocarbons. J. Mol. Model. 2013, 19, 2845-2848. 